\title{
AugerPrime: the upgrade program of the Pierre Auger Observatory.
}

\section{Gabriella Cataldi $^{* a}$ for the Pierre Auger Collaboration ${ }^{b}$}

a INFN, sezione di Lecce, via per Arnesano, 73100 Lecce, Italy

${ }^{b}$ Observatorio Pierre Auger, Av. San Martín Norte 304, 5613 Malargüe, Argentina

E-mail: auger_spokespersons@fnal.gov

Full author list: http://www.auger.org/archive/authors_2017_06.html

\begin{abstract}
The highest-energy cosmic rays measured with the Pierre Auger Observatory over the past decade provide us with unprecedented glimpses into their origin and properties. Improvements to this understanding will come from a major upgrade stage of the Observatory denominated AugerPrime. The upgrade program will include new plastic scintillator detectors on top of all water-Cherenkov detectors, faster and more powerful electronics, and the extension of the dynamic range. Complementing the water-Cherenkov detectors of the surface array with scintillator detectors, we will be able to evaluate the muonic component of the showers better and thus to estimate the primary mass of the ultra high energy cosmic rays on an event by event basis, reaching the region of the flux suppression. The enhanced information on composition will allow us to perform anisotropy studies on selections of mass groups and to explore the hadronic interactions in an unexplored energy and kinematic region. After introducing the physics motivation for upgrading the Auger Observatory, the planned detector upgrade and the technical realization will be presented, with an emphasis on the expected performance and the improved physics sensitivity.
\end{abstract}

The European Physical Society Conference on High Energy Physics

5-12 July, 2017

Venice

${ }^{*}$ Speaker. 


\section{Introduction}

The investigation of the data set gathered with the Pierre Auger Observatory[1] has resulted in major progress in the field of ultra-high energy cosmic rays (UHECRs), but a coherent understanding has not yet been accomplished [2]. An upgrade of the Auger Observatory (named AugerPrime $[3,4]$ ) has been arranged to broaden the composition sensitivity of the Observatory into the flux suppression region of the spectrum. The major point of AugerPrime is to derive the primary mass of the highest energy cosmic rays on a shower-by-shower premise, providing supplementary measurements of composition-sensitive observables. The search for the origin of the flux suppression will set key limits on the possible astrophysical sources and will enable us to determine more accurate estimates of gamma-ray and neutrino fluxes at ultra-high energy. The measurement of the flux contribution of protons will enhance the physics capability of existing and future cosmic ray, gamma-ray and neutrino detectors. With such a specific end goal, the aim of AugerPrime is to achieve a sensitivity of as little as $10 \%$, in the flux contribution of protons in the suppression region. The determination of the primary mass composition of ultra-high energy cosmic rays is profoundly connected with our comprehension of extensive air showers and hadronic interactions. In the Auger data, there is a controversy between the observed and expected muon numbers, therefore it is of fundamental importance to examine the hadronic multiparticle production in extensive air showers.

\section{The Detector Upgrade}

The AugerPrime upgrade is composed of many enhancements to the Pierre Auger Observatory. The most crucial is a thin scintillation detector (called the Surface Scintillator Detector (SSD)) positioned over, and triggered by, each of the existing water-Cherenkov detectors (WCD).

An SSD unit consists of a box of area $3.8 \mathrm{~m} \times 1.3 \mathrm{~m}$, containing two scintillator sub-modules, each composed of 24 bars of extruded scintillator bars of about $1.6 \mathrm{~m}$ length, $5 \mathrm{~cm}$ width and $1 \mathrm{~cm}$ thickness. The $3.8 \mathrm{~m}^{2}$ scintillator planes are housed in light-tight, weatherproof enclosures, attached to the existing WCD with a strong support frame (see Figure 1). The scintillator light is read out with wavelength-shifting fibres (Kuraray Y11(300)M S-type) inserted into straight extruded holes in the scintillator planes, which are bundled and attached to a single photomultiplier tube (PMT). As PMT the 8-stage, 38mm diameter Hamamatsu R9420 has been selected as baseline design. It has a bi-alkali photo-cathode, with a quantum efficiency of about $18 \%$ at the wavelength of $500 \mathrm{~nm}$ and a very linear response.

The other essential improvement introduced in the AugerPrime program is the redesign of the electronics of the WCD and the increase of its dynamic range (three times the present). The new electronics will process both WCD and SSD signals that will be sampled synchronously at a rate of $120 \mathrm{MHz}$. Moreover the data will take advantage of better timing accuracy and faster ADC sampling. A new GPS receiver will permit a timing accuracy of 5 nanoseconds, about a factor two superior to the present system. The use of a more powerful processor and FPGA allows better data processing and the setup of more advanced local triggers. The dynamic range of the WCD will be improved by a factor 32 with an additional small (1") PMT that will be inserted in the WCD. 

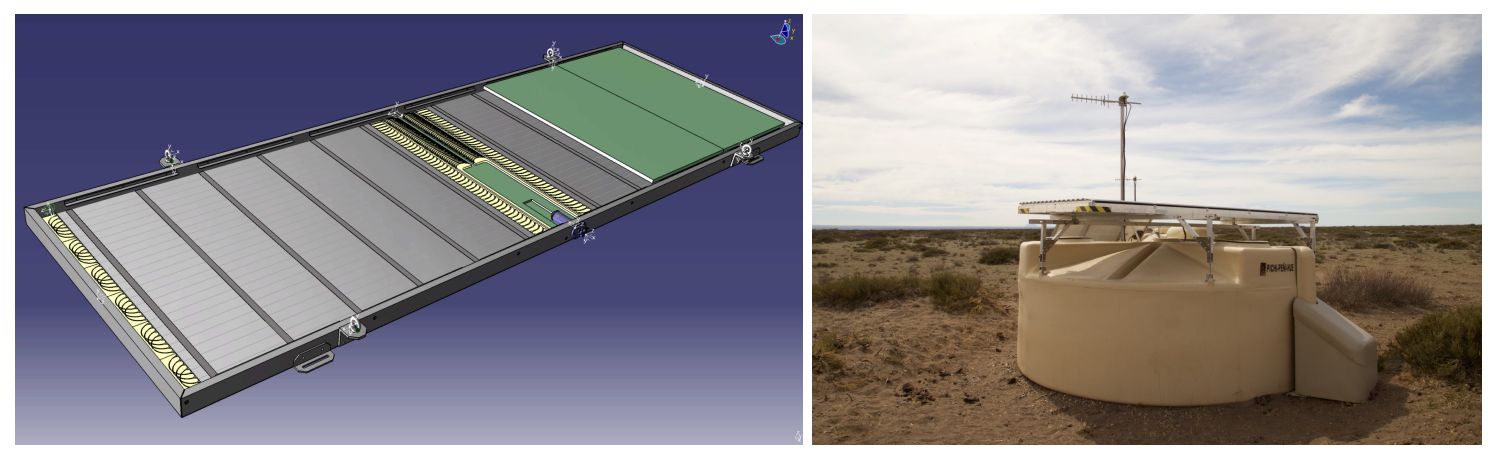

Figure 1: Left: The design of the Surface Scintillator Detector (SSD); Right: One station of the AugerPrime Engineering Array.

To confirm and calibrate the techniques used to separate shower muon content utilizing the SSD and WCD stations, an underground muon identifier (AMIGA) will be introduced to give an immediate estimation of the muon content of a sample of showers observed by the SSD [5].

The Auger Fluorescence Detector (FD) [6] provides information about extensive air showers such as a model-independent energy reconstruction and their longitudinal development profiles. The main limitation of the FD is its duty cycle, as of now at the level of $15 \%$. A significant increase of the duty cycle is conceivable by the extension of the FD operation to times at which a larger fraction of the moon in the sky is bright. During such operating conditions the PMT gains must be compensated by bringing down the supplied high voltage to avoid a high anode current that will produce a deterioration of the PMTs. The HV power supplies utilized for the FD permit exchanging between two high voltage levels and the PMTs can be operated at the nominal gain (standard operation mode) and at a lower gain (new operation mode for high night sky background).

\section{Science Impact of the Upgrade}

The density of muons is the most direct composition-sensitive and model-independent observable to be obtained from the upgraded detector array. The signal responses to particles of the muonic and electromagnetic shower components in the two detectors (SSD and WCD) enable one to infer the muonic signal on a single station premise. This can be understood by comparing the signal for different shower components as shown in Figure 2. Over a wide range in lateral separation, the ratio between the integrated signal of electromagnetic particles (photons and electrons) and that of muons is more than a factor two higher in an unshielded scintillation detector compared with a WCD [4].

One of the fundamental purposes of the physics capacity of the Auger Upgrade is that of being capable to contradistinguish different composition and, accordingly, physics scenarios in the energy range of the flux suppression. This is troublesome to establish without an a priori knowledge of composition. Thus two benchmark spectra have been picked as portrayals of a maximum-rigidity scenario (scenario 1) and a photo-disintegration scenario (scenario 2), as explained in [7].

Figure 3 shows the mean depth of shower maximum $\left(X_{\max }\right)$ and the corresponding $\sigma\left(X_{\max }\right)$ for these scenarios, using only the data of the upgraded observatory stations (Surface Detector). The 

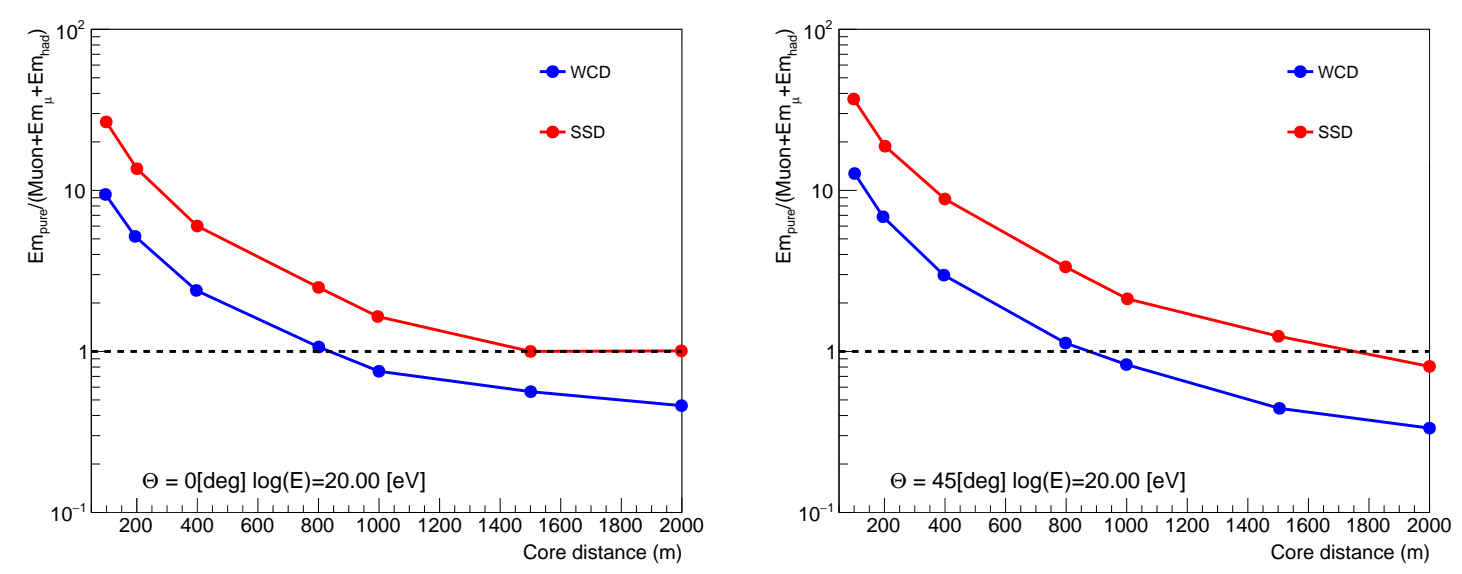

Figure 2: Ratios between the electromagnetic component and the muonic component of different contributions to the integrated signal detected for air showers of $10^{20} \mathrm{eV}$ at two zenith angles. The label"WCD" corresponds to the Water-Cherenkov Detectors, and the "SSD" to the surface scintillator detectors.
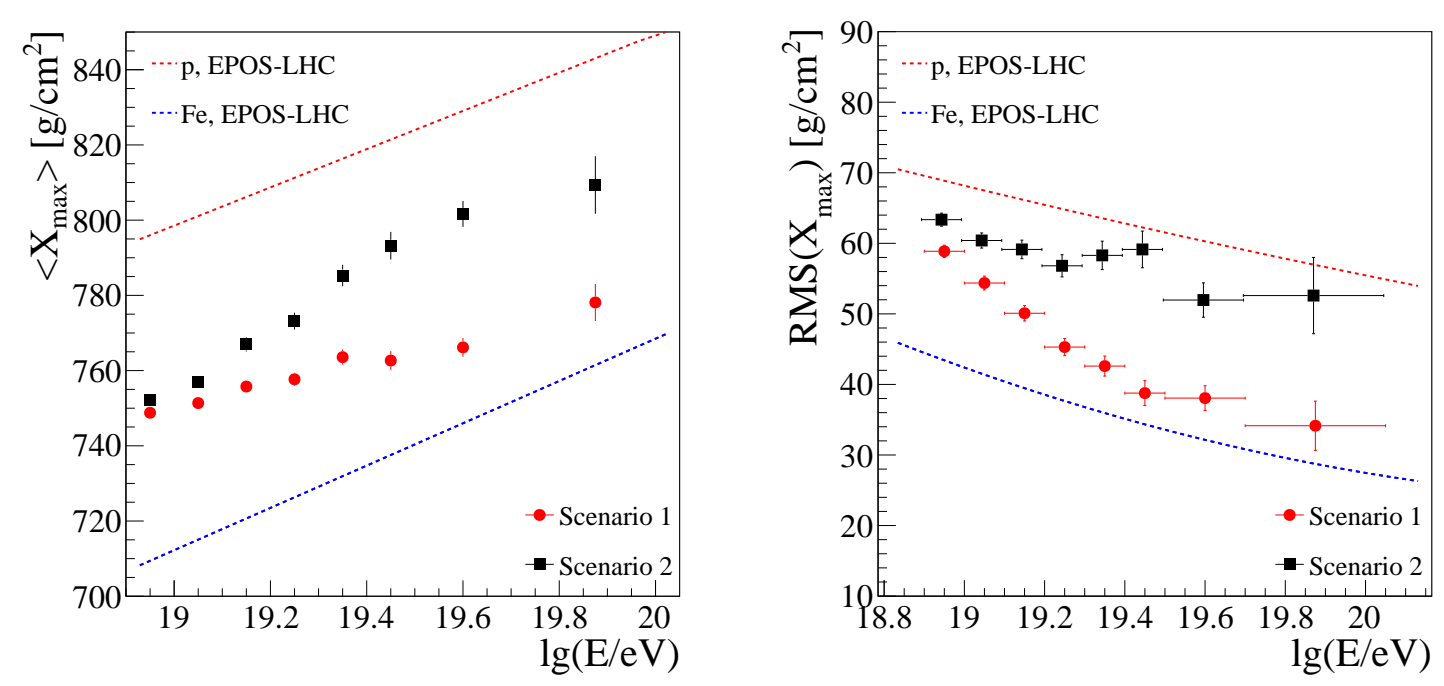

Figure 3: Predicted $X_{\max }$ and $\sigma\left(X_{\max }\right)$ for the two benchmark scenarios ( Scenario 1: maximum-rigidity; Scenario 2: photo-disintegration

$\sigma\left(X_{\max }\right)$ encloses the intrinsic air-shower fluctuations and the detector resolution. In the Figure are shown also the expectations for pure proton and pure iron compositions.

The mean $X_{\max }$ and $\sigma\left(X_{\max }\right)$ are comparable for the two scenarios up to $10^{19.2} \mathrm{eV}$. This is the energy range included in the data of the fluorescence telescopes. Going forward into the suppression region, the models foresee significantly different extrapolations and the two scenarios can be distinguished with high significance and statistics. Moreover, the availability of the muon information event-by-event allows studies of the aspects of hadronic interactions and will allow the selection of events in the cutoff region enriched with light elements, improving the capability of the Observatory to identify the possible sources of UHECRs. 

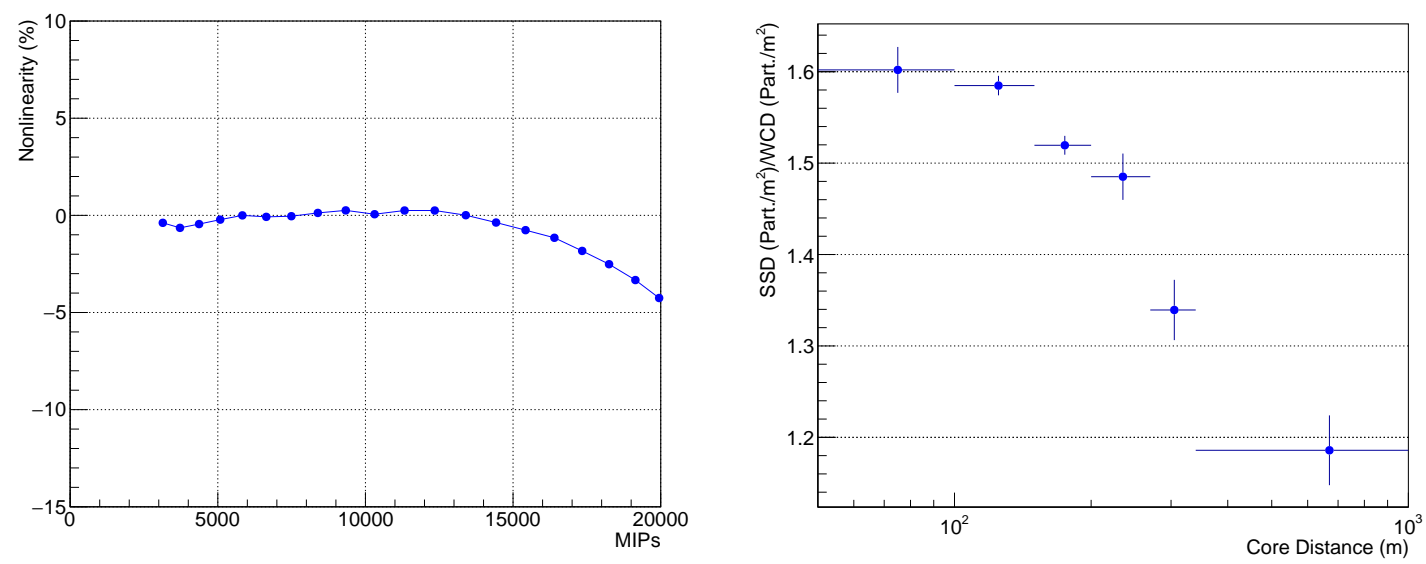

Figure 4: Left: Measured dynamic range of one SSD detector, the detector is linear within 5\% up to 16000 particles. Right: ratio between the SSD and the WCD particle density versus the distance from the shower core (detectors without signals have been excluded)

\section{Status of the Upgrade}

The first twelve stations of the Engineering Array(EA) of AugerPrime were assembled in Europe and deployed in the Pierre Auger Observatory in September 2016. A part (9 detectors) of the EA is located inside the standard $1500 \mathrm{~m}$ array while the rest of the stations are in the more dense area of the $750 \mathrm{~m}$ spaced array. The EA has been in continuous data taking mode since October 2016 and has collected more that 30000 local triggers.

The performance of the upgraded stations has been monitored with the data collected by the EA. The upgraded stations produce signals that are in good agreement with expectations.

Crucial to the measurement of primary mass with the upgraded stations are the detectors viewing a large number of particles. To avoid saturated stations close to the shower core a strong effort in the upgrade has been dedicated to the extension of the dynamic range of the WCD and in providing an acceptable dynamic range for the SSD. In Figure 4 (left) is shown the measured dynamic range of one of the SSD stations in the EA. The figure shows that the SSDs are linear within 5\% up to a signal of 20000 particles. The SSDs are more sensitive to the electromagnetic component of the extensive air showers. The lateral density function (LDF) of SSD signals is higher than the corresponding LDF of the WCD in the region close to the shower axis (see Figure 4 (right)). Far from the shower core the showers are dominated by the muons and the signal density in the two detectors is similar. The different responses of the two detectors to the two main components of extensive air showers is the key tool to identify the mass of the primary cosmic rays on an event-by-event premise [4].

\section{Conclusions}

AugerPrime promises high-quality future data. With operation planned from 2018 until 2024, event statistics will more than double compared with the existing Auger data set, with the critical added advantage that every event will have mass information and will allow a better addressing of 
some of the most pressing questions in UHECR physics. The use of composition-sensitive information will help to better reconstruct the properties of the primary particles at the highest energies, and will improve the measurements in the important energy range just above the spectral ankle. Furthermore, measurements with the new detectors will help to reduce systematic uncertainties related to modeling hadronic showers and to the restrictions of reconstruction algorithms. This enhanced knowledge of air-shower physics will permit a re-analysis of existing data for improved energy assignments, for mass composition studies, and for photon and neutrino searches. A new agreement has been signed between the funding agencies in November 2015 extending the acquisition period of the Pierre Auger Observatory until 2024 and supporting the AugerPrime upgrade. The start of the deployment of the upgraded stations is expected in January 2018.

\section{References}

[1] A. Aab et al. [Pierre Auger Coll.], The Pierre Auger Cosmic Ray Observatory, Nucl. Instrum. Meth. A798 (2015) 172.

[2] I. Lhenry-Yvon, for the Pierre Auger Collaboration. [Pierre Auger Coll.], Latest results of the Pierre Auger Observatory, in these proceedings.

[3] A. Aab et al. [Pierre Auger Coll.], Upgrade of the Pierre Auger Observatory (AugerPrime), in Proceedings of "34th International Cosmic Ray Conference" PoS(ICRC2015)686.

[4] A. Aab et al. [Pierre Auger Coll.], The Pierre Auger Observatory Upgrade AugerPrime: Preliminary Design Report, in arXiv:1604.03637 [astro-ph.IM] (2015).

[5] A. Aab et al. [Pierre Auger Coll.], Prototype muon detectors for the AMIGA component of the Pierre Auger Observatory, Journal of Instrumentation, 11 (2016) P02012 .

[6] J. Abraham et al. [Pierre Auger Coll.], The Fluorescence Detector of the Pierre Auger Observatory, Nucl. Instrum. Meth. A620 (2010) 227.

[7] A. di. Matteo et al. [Pierre Auger Coll.], Combined fit of spectrum and composition data as measured by the Pierre Auger Observatory, in Proceedings of "34th International Cosmic Ray Conference" PoS(ICRC2015)249. 